\title{
Computational Model of a Pacinian \\ Corpuscle for an Electrical Stimulus: Spike-Rate and Threshold Characteristics
}

\author{
Madhan Kumar Vasudevan ${ }^{(\bowtie)}$, Rahul Kumar Ray, \\ and Manivannan Muniyandi \\ Touch Lab, Department of Applied Mechanics, \\ Indian Institute of Technology Madras, Chennai 600036, India \\ madhan.kv@gmail.com, rahulraiecb@gmail.com, mani@iitm.ac.in
}

\begin{abstract}
Understanding the response of Pacinian Corpuscle (PC) for an electrical stimulus through a computational model can give better insight into the physiology. Although there are simpler models available in the literature, models simulating spike-rate and threshold characterizations are still missing. These characterizations may lead to the development of tactile displays combining both electrical and mechanical stimuli, especially high-frequency vibrations. We developed a PC model with equivalent circuits of the electrode-skin interface, PC's neurite, and the first Ranvier node. The input electrical stimulus is a current pulse with varying amplitude (0 to $2 \mathrm{~mA}$ ) and varying frequency $(5 \mathrm{~Hz}$ to $1600 \mathrm{~Hz})$. The model is characterized initially for the frequency response, and then the spike-rate and threshold characteristics were simulated. The spike-rate traces for electrical stimuli show the phase-locking phenomenon similar to the mechanical stimuli responses of PC, however the plateau lengths are larger for the spike-rate traces with electrical stimuli compared to that of the mechanical stimuli. This is reflected as a large difference in the threshold characteristics for one and two impulsesper-cycle. Moreover, threshold characteristics are little influenced by the neural noise. This model can be extended to study the combination of electrical and mechanical stimuli.
\end{abstract}

Keywords: Electrical stimulation $\cdot$ Neurite $\cdot$ Ranvier node $\cdot$ Spike-rate $\cdot$ Threshold.

\section{Introduction}

The electrical stimulus applied over the skin elicits different sensations such as continuous or intermittent touch depending on the frequency, phase, and amplitude of the stimulus [14]. The variations in the electrical stimulus can induce the receptors of various modalities such as mechano-, chemo-, and thermoreceptors. These variations are then decoded by the CNS to perceive various aspects of touch and other sensations [15]. Pacinian corpuscle (PC) being the most sensitive mechanoreceptor in the human body is responsible for the sensation of

(C) The Author(s) 2020

I. Nisky et al. (Eds.): EuroHaptics 2020, LNCS 12272, pp. 203-213, 2020.

https://doi.org/10.1007/978-3-030-58147-3_23 
high-frequency vibrations. PC neurite and the axon can be selectively stimulated by an electrical stimulus to induce the sensation of high-frequency vibration. To understand the physiology of a PC for mechanical or electrical stimuli, it is necessary to understand the morphology of a PC in finer details $[6,6,7,11,22,25]$ and the computational models [3,19,23,26,27].

The PC is made of an onion-like lamellar structure filled with interlamellar fluid [22]. The lamellar structure of the capsule acts as a mechanical band-pass filter $[9,19]$, which is the main reason for the rapid adaptiveness of the PC for a mechanical stimulus. Although the lamellar structure of the PC helps it to be the most rapidly adaptive mechanoreceptor, the neurite membrane and inner core also contribute to its rapid adaptiveness [1]. Due to the presence of stretchactivated and voltage-activated ion channels (SAICs and VAICs) in PC neurite [21], the applied mechanical stimulus gets converted into electrical spikes. Since lamellae are attached to each other by tight junctions, fluid in the inner core is considered to be electrically isolated from that in the outer core [12]. Moreover, the outer core is electrically isolated from the transductive portion of the neurite [1].

The exact location of action potential generation is still in controversy. It was believed that the core of the PC is rigid $[17,19]$, however, it was discovered later that the neurite of the PC contains SAICs and VAICs [1]. Models were developed, assuming that the action potential spikes from the first Ranvier node [14]. The controversy related to the site of initiation of action potential inside the PC capsule started from the discovery of the generator or local potential of PC inside the capsule [10]. Based on the majority of the evidence, it appears that the action potential at 1st Ranvier's node can be generated by the direct electrical stimulation at the nerve fiber [1].

\subsection{Motivation and Objective}

From the literature, it is clear that the characterization of a PC model for electrical stimuli in terms of spike-rate and threshold characteristics is still missing. Neural spikes encode different features of the stimulus which include amplitude, frequency and even the location of the stimulus over the skin [27]. Spike rate and threshold characteristics of a neural spike train help in understanding how the stimulus features are conveyed to the CNS. Such characterizations would be useful in comparing the physiological characteristics of a PC stimulated electrically, and that of a PC stimulated mechanically. Our objective in this work is to model and characterize the response of a PC for an electrical stimulus applied over the skin. We characterize our model based on frequency response, spike-rate, and threshold characteristics.

\section{Method}

The computational model of a Pacinian corpuscle excited by electrical stimuli is described in this section. This model is an extension of our previous modeling 
work (BMS model) [2-4] for an isolated PC excited by mechanical stimuli into a PC model excited by electrical stimuli. The model includes the electrode-skin interface, neurite, and the first Ranvier node of a PC. The simulation involves the application of electrical stimuli of various frequencies and the characterization of the model for frequency response, spike-rate and threshold characteristics.

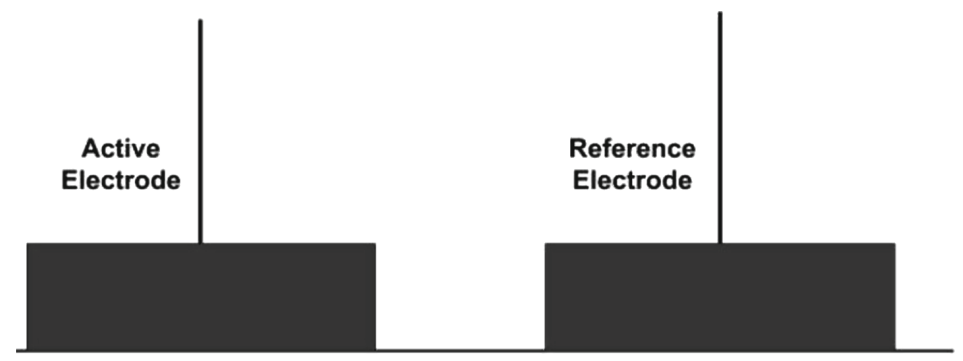

Epidermis

\section{Dermis}

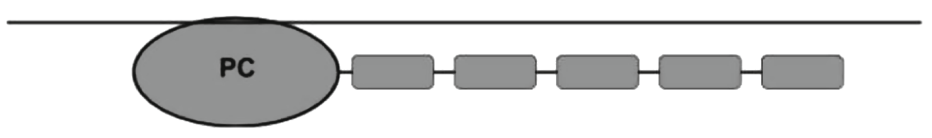

Fig. 1. Electrical stimulation of a Pacinian nerve through the skin surface. The stimulus current pulse is applied through a two-electrode electrical circuit. The axon of PC located deep within the skin gets excited by the current stimulus applied perpendicular to it. The frequency and amplitude of the current pulse can be varied for the measurement of a threshold of sensation.

\subsection{Model Description}

The electrode-skin interface, PC's neurite, and the first Ranvier node are modeled using electrical parameters available from Chan [8], Saadi et al. [24], Khorshid et al. [16] and Biswas et al. [2]. The conceptual depiction of the proposed model is shown in Fig. 1. The electrical current pulse is applied over the skin through active and reference electrodes. PC has a myelinated axon and it is located deep within the skin compared to other mechanoreceptors [6]. In our model, we assume that the axon of the PC is perpendicular to the application of an electrical stimulus. The applied electrical stimulus gets filtered by the skin layers, and then the filtered stimulus undergoes two-stage non-linear neural-spike generation. This spike generation process is modeled as an Adaptive Relaxation Pulse Frequency Modulator (ARPFM) which was introduced by Biswas et al. [4]. The ARPFM is similar to the integrate-and-fire neuron model except that 


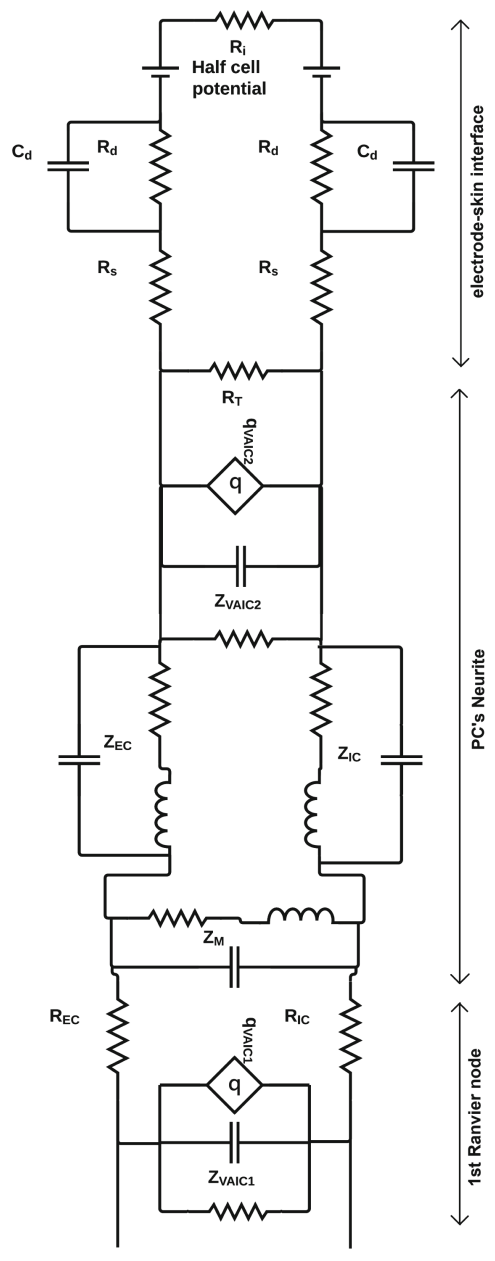

(a)

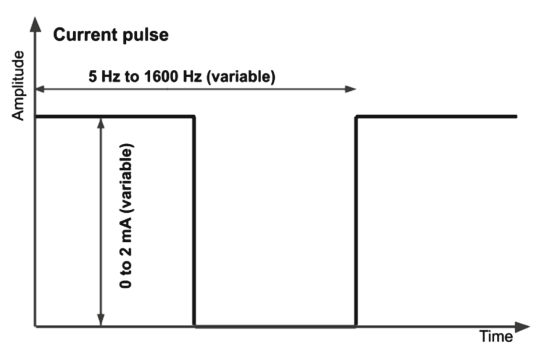

(b)

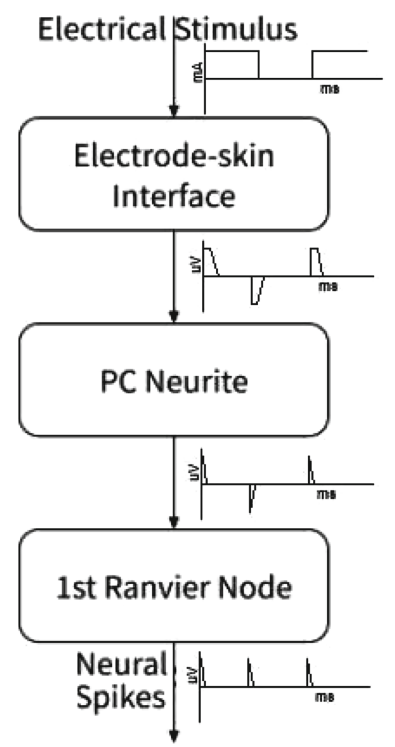

(c)

Fig. 2. (a) Equivalent circuit model of the electrode-skin interface, PC's neurite, and the first Ranvier node. The values of potential and impedance are adapted from $[2,8$, 16,24]. (b) Current pulse with variable amplitude and frequency, and with fixed duty cycle $(50 \%)$. (c) Block diagram of a PC model with a representation of signals at each level.

the integrator is lossy, and the threshold is adaptive. The neural noise for the ARPFM stage is modeled as an additive random noise and multiplied with the adaptive threshold for the spike generation.

The proposed model of a PC for electrical stimulation is shown in Fig. 2. It consists of three stages, as shown in Fig. 2c, electrode-skin interface model, PC's neurite model, and the first Ranvier node model. Each of them is modeled with electrical components whose parametric values are adapted from Chan [8], 
Saadi et al. [24], Khorshid et al. [16] and Biswas et al. [2]. The electrode-skin interface model, as shown in Fig. 2a (I), consists of tissue impedance $R_{T}$, skin resistance $R_{s}$, double layer impedance $R_{d}, C_{d}$, and half cell potential. Since there are two electrodes, active and reference, the same model is mimicked for both. That is, the electrical properties and parameters of both electrode-skin interfaces are assumed to be identical. The skin impedance measurement reviewed in $\mathrm{Lu}$ et al. [20] explains that the electrical impedance of the skin is mainly due to stratum corneum (the uppermost layer of the epidermis) and the impedance of the other layers is comparatively low. The impedance function is given as

$$
K(s)=\frac{0.0259 s+1859}{0.01858 s+1}
$$

The second stage of the model contains a circuit equivalent of the PC's neurite as shown in Fig. 2a (II). This stage introduces non-linearity in the generation of the receptor potential. It consists of a non-linear dependent charge source $q_{\mathrm{VAIC} 2}$ and its impedance $Z_{\mathrm{VAIC} 2}$. The impedances of extra-cellular $Z_{\mathrm{EC}}$ and intra-cellular $Z_{\mathrm{IC}}$ matrices are also included in the model along with impedance of axolemma membrane $Z_{\mathrm{M}}$. The PC's neurite parameters and the values of impedances are the same as given in Biswas et al. [2]. The output of the second stage is the receptor potential. It is assumed that the receptor potential from the second stage is the input to the third stage. The third stage of the model includes the equivalent circuit of the first Ranvier node, as shown in Fig. 2a (III). This circuit contains a non-linear dependent charge source $q_{\mathrm{VAIC1}}$ and the impedance parameter $Z_{\mathrm{VAIC} 1}$, both models the VAIC located in the first Ranvier node. The ARPFM threshold is actually a measure of the refractoriness of the VAICs to go for the next avalanche opening. In comparison to the model of VAIC, as ARPFM, the threshold is adaptive and amplified by the threshold amplification factor in the refractory period, as found in Loewenstein and Altamirano-Orrego [18]. It is observed that after the time $(\mathrm{t})=2.5 \mathrm{~ms}$, the experimental data have an exponential decay with a time constant of $0.56 \mathrm{~ms}$. The threshold amplification factor (TAF) considered for the ARPFM [4] is given as

$$
T A F=1+\left(7.75 * t^{-0.16} * \exp (-0.56 t)\right)
$$

The electrical stimulus is shown in Fig. 2b, which has variable amplitude from 0 to $2 \mathrm{~mA}$ and variable frequency from $5 \mathrm{~Hz}$ to $1600 \mathrm{~Hz}$. The duty cycle of the applied electrical stimulus is always $50 \%$.

Model Parameters: The model parameters for the PC model are same as given in $[3,19]$ and also the parameters and approximations for the electrodeskin interface model are same as given $[8,16,24]$.

$$
\begin{aligned}
R_{i}, R_{d}, R_{s}, R_{T}: & \text { Resistance of electrical stimulation circuit, double layer, skin } \\
& \text { and tissue, respectively } \\
C_{d}: \text { Double layer capacitance } & \text { : }
\end{aligned}
$$

$q_{\mathrm{VAIC} 2}, q_{\mathrm{VAIC1}}$ : Voltage activated ion channels in neurite (2) and 1st Ranvier node (1) are modeled as voltage dependent charge sources. 
$Z_{E C}, Z_{I C}, Z_{M}$ : Impedance of extracellular matrix, intracellular matrix and membrane of axolemma, respectively.

$K(s)$ : Transfer function of electrode-skin interface model in Laplace domain.

Model Approximations: The following are the approximations assumed for the developed model,

a) We consider only one type of mechanoreceptor under the skin, the PC, although the Meissner is also a rapidly adaptive receptor.

b) The axon of a PC is perpendicular to the direction of the electrical stimulus.

c) The half cell potential of the electrode-skin interface model is assumed to be identical. Moreover, elements of the electrode-skin interface model for both the electrodes are identical, according to [8].

d) All the simulations are limited to only the vibrotactile stimulus with the frequency ranging from $5 \mathrm{~Hz}$ to $2000 \mathrm{~Hz}$ with $50 \%$ duty cycle.

\section{Results and Discussion}

The objective of this work is to develop a PC model for an electrical stimulus and characterize the model for frequency response, spike-rate and threshold characteristics.

\subsection{Frequency Response}

Figure $3 \mathrm{a}$ shows the bode plot of the electrode-skin-PC model, including the electrode-skin interface, PC's neurite, and the first Ranvier node. It may be observed that the overall model is a typical high-pass filter. The slope of the magnitude plot changes significantly; from $1 \mathrm{~Hz}$ to $10 \mathrm{~Hz}$ it is $\approx 10 \mathrm{~dB} /$ decade, from $10 \mathrm{~Hz}$ to $10 \mathrm{kHz}$ it is $\approx 20 \mathrm{~dB} /$ decade and from $20 \mathrm{kHz}$ the slope approaches zero. On the other hand, the phase difference is found to be within 90 to $45^{\circ}$ for the frequencies from $1 \mathrm{~Hz}$ to $10 \mathrm{kHz}$, reaching its peak phase shift of $\approx 90^{\circ}$.

The bode plot shown here can be compared to that of the PC model with a mechanical stimulus [3]. Although the magnitude plot is almost the same as that of the PC model with a mechanical stimulus, the phase plot differs slightly for frequencies lesser than $5 \mathrm{~Hz}$. Since the present model focuses on frequencies of more than $5 \mathrm{~Hz}$, this small difference may be ignored. Also, at this low frequency other mechanoreceptors respond better than the PC [15]. 


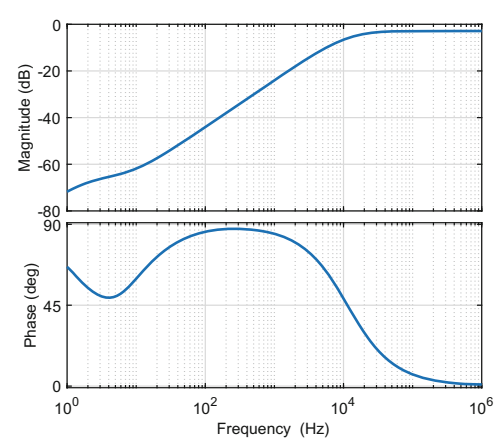

(a)
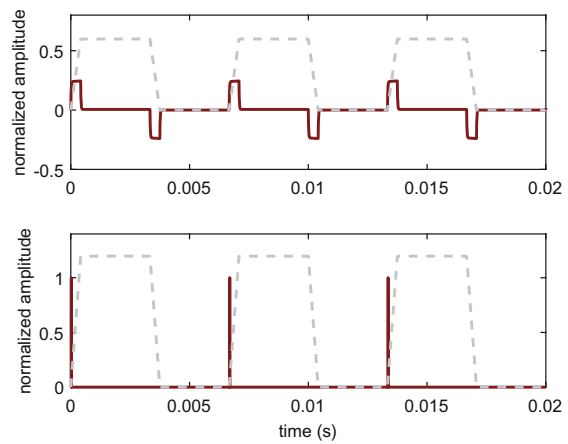

(b)

Fig. 3. (a) Magnitude response (top) and phase response (bottom) of the PC model for electrical stimuli. The magnitude in (a) indicates the gain in $\mathrm{dB}$ with respect to $1 \mathrm{~mA}$ electrical stimulus. (b) The response of the electrode-skin interface model (top), the spike response at the output of the first Ranvier node (bottom). The dashed lines in (b) indicate the applied electric stimulus as shown in Fig. 2b. All the amplitudes are normalized for the representation. The ordinate unit for dashed lines is $\mathrm{mA}$ and that for the solid lines is in $\mu V$.

\subsection{Spike-Rate Characteristics}

The spike response of the developed model is shown in Fig. 3b. The electrical stimulus that gets filtered by the electrode-skin interface model reaches the PC's neurite then the first Ranvier node to fire the neural spikes, as shown in Fig. 3b. The response shown here is the typical one impulse-per-cycle (ipc) response for the applied electrical stimulus.

The spike-rate versus stimulus amplitude plots for various stimulus frequencies are shown in Fig. 4a and 4c. We have also shown the spike-rate plots generated by a PC model for a mechanical stimulus in Fig. 4b and 4d (images adapted from Biswas et al. [4]), which are given adjacently for comparison. For each stimulus frequency of Fig. 4a, the spike-rate is zero until the stimulus reaches a certain threshold. Once the threshold is reached, it increases steeply and reaches a series of plateaus at spike-rates that are multiples of stimulus frequency. This phenomenon is known as phase-locking [5]. For instance, a $50 \mathrm{~Hz}$ stimulus gets plateaued during 50 Spikes Per Second (sps), 100 sps, 150 sps and 200 sps which are consistent with one, two, three and four impulse-per-cycles respectively according to Johnson [13] who recorded the population response from median nerves of 26 monkeys. This phase-locking, non-linear jumps, and plateau of the spike-rates depend on the stimulus frequency, as mentioned in [5]. The spike-rate gets saturated for $800 \mathrm{~Hz}$ and $1600 \mathrm{~Hz}$ at around 1000 sps.

Unlike the spike-rate plot for mechanical stimulus as given in Fig. 4b which contains short plateau lengths, the spike rate plot for electrical stimulus contains longer plateaus. These longer plateaus are reflected in the threshold characteristics as well which we are discussing subsequently in the next subsection. 


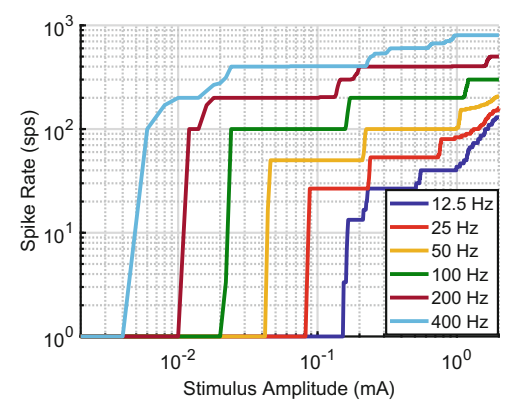

(a)

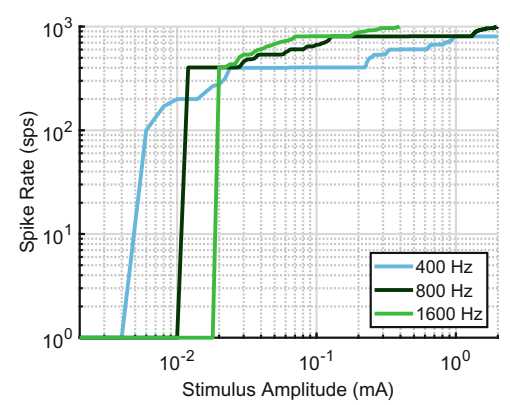

(c)

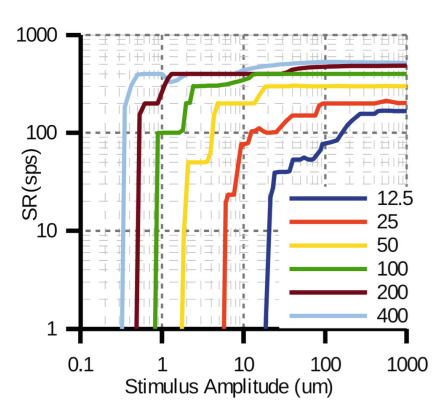

(b)

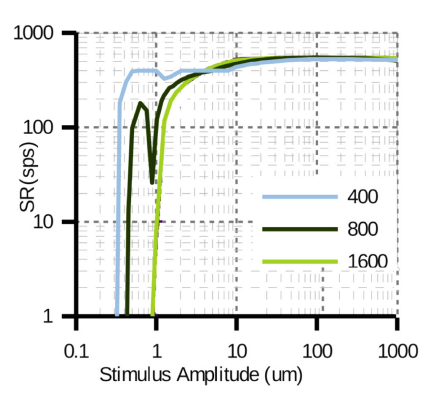

(d)

Fig. 4. (a) and (c) represents the Spike-rate (sps - number of spikes per second) characteristics plotted for the variation in stimulus amplitude (0 to $2 \mathrm{~mA})$ with various constant stimulus frequencies. (b) and (d) represents the same for mechanical stimuli (image adapted from Biswas et al. [4]). Although the spike-rate characteristics for electrical stimuli exhibit a phase-locking phenomenon [5], the plateau lengths are larger compared to that of the characteristics in the mechanical stimuli model.

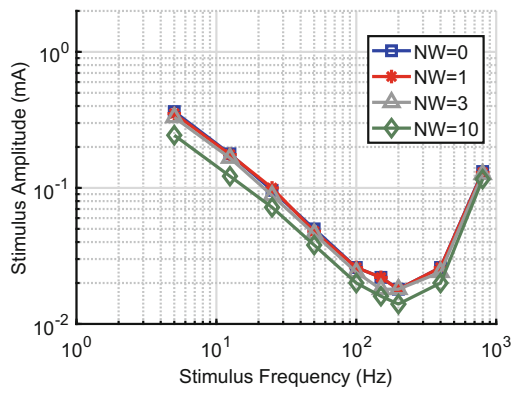

(a)

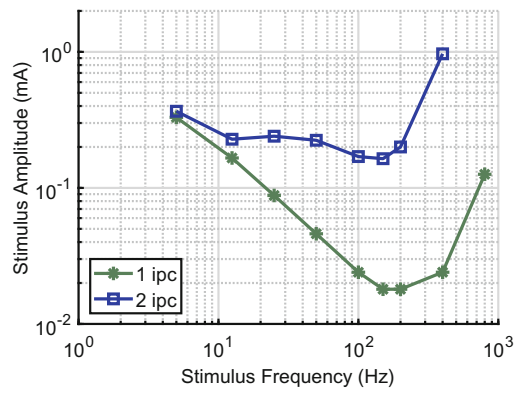

(b)

Fig. 5. (a) Stimulus amplitude versus stimulus frequency, for various noise weights. Except for $\mathrm{NW}=10$, we can observe that there is only little influence of noise weights in the threshold. (b) Minimum stimulus amplitude required to elicit one and two impulseper-cycle (ipc) for varying stimulus frequency. This plot can be compared with the plataeu lengths of Fig. 4 a to understand the shift in 2 ipc from 1 ipc curve here. 
Physiologically, longer plateaus may be due to the various levels of saturation in the VAIC characteristics of the first Ranvier node induced by the electrical stimuli. Moreover, both the electrical and mechanical stimuli spike rate plots in Fig. 4 contain varying thresholds for each stimulus frequency to initiate spikes. This can be correlated with the increasing magnitude response of the model, as shown in Fig. 3a. Moreover, we can observe the saturation of spike-rate traces for the stimulus frequencies greater than $400 \mathrm{~Hz}$ as shown in Fig. $4 \mathrm{~d}$ around $600 \mathrm{sps}$, whereas in Fig. 4c the saturation occurs only after 1000 sps.

\subsection{Threshold Characteristics}

The threshold characteristics simulated in this model are for one impulse-percycle (ipc) for various neural noises, which is shown in Fig. 5a. We can observe that the model response is not much influenced by the neural noise, except for noise weight $(\mathrm{NW})$ of 10 . The threshold characteristics simulated here may be considered as consistent with the well known psychophysical VPT curve [28] and the lowermost threshold is achieved for $200 \mathrm{~Hz}$ electrical stimulus. Moreover, the minimum stimulus amplitude required to elicit one and two impulse-per-cycle (ipc) for varying stimulus frequency is plotted and shown in Fig. 5b. We can observe that each trace of the threshold curve is unique and further away. This may be due to the longer plateaus as shown in Fig. 4.

\subsection{Possible Extensions}

In this paper, we assumed that the electrical stimulus reaches the first Ravier node only through the PC neurite, not directly. A special case can be considered for this model in which the applied electrical stimulus reaches both PC's neurite and the first Ranvier node simultaneously. In this case, input to the first Ranvier node will be the sum of the output from PC's neurite (receptor potentials) and the electrical stimulus itself, both acting together to reach the threshold in the Ranvier node. Although this special case is not explicitly shown in the model figures, it can also be simulated from the same model by simple addition, as mentioned here. The model can also be simulated for various duty cycles of electrical stimuli whereas in the present work we have considered a fixed duty cycle of $50 \%$.

\section{Summary}

We developed a model for the response of Pacinian Corpuscle excited by an electrical stimulus. We adapted and combined the models of the electrode-skin interface, PC's neurite, and the first Ranvier node, and characterize them together for frequency response, spike-rate, and threshold characteristics. Although there are models known for the electrical stimulus to a PC, none of them explain in terms of the aforementioned characteristics. We have shown the frequency response using a bode plot, which shows constant gain after $10 \mathrm{kHz}$. The spikerate plots for varying stimulus amplitudes were simulated and the plateaus were 
observed for each stimulus frequencies. The threshold characteristics were simulated for one and two impulses-per-cycle (ipc). Furthermore, for a 1 ipc, the threshold characteristics were simulated for various noise weights. We compared our results with the existing models of PC for mechanical stimulus. The future work may include developing a PC model 1) for the combination of electrical and mechanical stimuli (hybrid stimuli) and perform psychophysical experiments to validate the model responses, and 2) for two different electrical stimuli of varying amplitude, frequency, and phase applied in two different locations over the skin to elicit various sensations.

\section{References}

1. Bell, J., Bolanowski, S., Holmes, M.H.: The structure and function of Pacinian corpuscles: a review. Prog. Neurobiol. 42(1), 79-128 (1994)

2. Biswas, A., Manivannan, M., Srinivasan, M.A.: A biomechanical model of Pacinian corpuscle \& skin. In: 2013 Biomedical Sciences and Engineering Conference (BSEC), pp. 1-4. IEEE (2013)

3. Biswas, A., Manivannan, M., Srinivasan, M.A.: Multiscale layered biomechanical model of the Pacinian corpuscle. IEEE Trans. Haptics 8(1), 31-42 (2015)

4. Biswas, A., Manivannan, M., Srinivasan, M.A.: Vibrotactile sensitivity threshold: nonlinear stochastic mechanotransduction model of the Pacinian corpuscle. IEEE Trans. Haptics 8(1), 102-113 (2015)

5. Bolanowski Jr., S., Zwislocki, J.J.: Intensity and frequency characteristics of Pacinian corpuscles. I. Action potentials. J. Neurophys. 51(4), 793-811 (1984)

6. Cauna, N., Mannan, G.: The structure of human digital Pacinian corpuscles (corpuscula lamellosa) and its functional significance. J. Anat. 92(Pt 1), 1 (1958)

7. Cauna, N., Mannan, G.: Development and postnatal changes of digital Pacinian corpuscles in the human hand. J. Anat. 93(Pt 3), 271 (1959)

8. Chan, A.Y.: Biomedical Device Technology: Principles and Design. Charles C Thomas Publisher, Springfield (2016)

9. Grandori, F., Pedotti, A.: A mathematical model of the Pacinian corpuscle. Biol. Cybern. 46(1), 7-16 (1982). https://doi.org/10.1007/BF00335347

10. Gray, J.A.B., Sato, M.: Properties of the receptor potential in Pacinian corpuscles. J. Phys. 122(3), 610-636 (1953)

11. Hubbard, S.: A study of rapid mechanical events in a mechanoreceptor. J. Phys. 141(2), 198-218 (1958)

12. Ide, C., Hayashi, S.: Specializations of plasma membranes in PCS: implications for mechano-electric transduction. J. Neurocytol. 16(6), 759-773 (1987)

13. Johnson, K.: Reconstruction of population response to a vibratory stimulus in quickly adapting mechanoreceptive afferent fiber population innervating glabrous skin of the monkey. J. Neurophysiol. 37(1), 48-72 (1974)

14. Kajimoto, H., Kawakami, N., Maeda, T., Tachi, S.: Tactile feeling display using functional electrical stimulation. In: Proceedings of the 1999 ICAT, p. 133 (1999)

15. Kandel, E., Schwartz, J., Jessell, T.: Principles of Neural Science. Prentice-Hall International Edit. Elsevier, Amsterdam (1991)

16. Khorshid, A.E., Alquaydheb, I.N., Eltawil, A.M.: Electrode impedance modeling for channel characterization for intra-body communication. In: Fortino, G., Wang, Z. (eds.) Advances in Body Area Networks I. IT, pp. 253-266. Springer, Cham (2019). https://doi.org/10.1007/978-3-030-02819-0_19 
17. Loewenstein, W.R.: The generation of electric activity in a nerve ending. Ann. N. Y. Acad. Sci. 81(2), 367-387 (1959)

18. Loewenstein, W.R., Altamirano-Orrego, R.: The refractory state of the generator and propagated potentials in a Pacinian corpuscle. J. Gen. Physiol. 41(4), 805-824 (1958)

19. Loewenstein, W., Skalak, R.: Mechanical transmission in a Pacinian corpuscle. An analysis and a theory. J. Physiol. 182(2), 346-378 (1966)

20. Lu, F., et al.: Review of stratum corneum impedance measurement in non-invasive penetration application. Biosensors 8(2), 31 (2018)

21. Pawson, L., Bolanowski, S.J.: Voltage-gated sodium channels are present on both the neural and capsular structures of Pacinian corpuscles. Somatosens. Mot. Res. 19(3), 231-237 (2002)

22. Pease, D.C., Quilliam, T.A.: Electron microscopy of the Pacinian corpuscle. J. Cell Biol. 3(3), 331-342 (1957)

23. Quindlen, J.C., Stolarski, H.K., Johnson, M.D., Barocas, V.H.: A multiphysics model of the Pacinian corpuscle. Integr. Biol. 8(11), 1111-1125 (2016)

24. Saadi, H., Attari, M.: Electrode-gel-skin interface characterization and modeling for surface biopotential recording: impedance measurements and noise. In: 2013 2nd International Conference on Advances in Biomedical Engineering, pp. 49-52. IEEE (2013)

25. Spencer, P.S., Schaumburg, H.H.: An ultrastructural study of the inner core of the Pacinian corpuscle. J. Neurocytol. 2(2), 217-235 (1973). https://doi.org/10.1007/ BF01474721

26. Summers, I.R., Pitts-Yushchenko, S., Winlove, C.P.: Structure of the Pacinian corpuscle: insights provided by improved mechanical modeling. IEEE Trans. Haptics 11(1), 146-150 (2018). https://doi.org/10.1109/TOH.2017.2769648

27. Vasudevan, M.K., Sadanand, V., Muniyandi, M., Srinivasan, M.A.: Coding source localization through inter-spike delay: modelling a cluster of Pacinian corpuscles using time-division multiplexing approach. Somatosens. Mot. Res. 37(2), 63-73 (2020)

28. Verrillo, R.T., Fraioli, A.J., Smith, R.L.: Sensation magnitude of vibrotactile stimuli. Perc. Psychophys. 6(6), 366-372 (1969). https://doi.org/10.3758/BF03212793

Open Access This chapter is licensed under the terms of the Creative Commons Attribution 4.0 International License (http://creativecommons.org/licenses/by/4.0/), which permits use, sharing, adaptation, distribution and reproduction in any medium or format, as long as you give appropriate credit to the original author(s) and the source, provide a link to the Creative Commons license and indicate if changes were made.

The images or other third party material in this chapter are included in the chapter's Creative Commons license, unless indicated otherwise in a credit line to the material. If material is not included in the chapter's Creative Commons license and your intended use is not permitted by statutory regulation or exceeds the permitted use, you will need to obtain permission directly from the copyright holder. 